\title{
Issues encountered in community health in Sri Lanka with special reference to Polpithigama rural village in North-Western province
}

\author{
Mapa Thilakarathna *
}

Faculty of Social Sciences, University of Kelaniya, Colombo, Sri Lanka

\author{
Key Words: \\ Community health \\ Community development \\ Chronic diseases
}

Received: 13 September 2017

Accepted: 10 October 2017

Published: 16 October 2017

\begin{abstract}
Community Health remains substantially significant in the Sri Lankan development sector as it concentrates much on community development for many decades. This study explores the existing issues of the rural community health sector, with special reference to a selected Grama Niladhari Division (GND) in North-Western province in Sri Lanka. Even though the rural development has much-focused community development projects, community health has not been sufficiently developed by the national and regional development sectors. Having conducted a field survey consisting of both qualitative in-depth interviews (10) and a general questionnaire survey (50), this study can be concluded that the majority of the subjects were not sufficiently aware of their health and hygiene by the respective community health programs conducted in this area for many years. Though there were many rural development projects in this area for many years, community health issues of identifying their health matters, chronic diseases, reproductive health risks, and sexually transmitted diseases were not substantially addressed by the respective community development programs.
\end{abstract}

\section{INTRODUCTION}

"Prevention is better than cure" signifies the importance of the existence of non-diseased human well-being than diseased by many chronic and non-communicative as well as all the currently widely spreading illnesses among the world. Developed countries as well as non- developed/developing countries are immensely applying Health Communication for a better society of disease and illness, albeit health communication emerged from the developed countries at the initial stage in mid-twentieth century. Behavioral Change Communication is another stream that applies communication for making a news-effective attitude and thereafter behaviour as a result of productive use of communication.

However, as an effect of globally overwhelming concern on the use and application of health communication for emerging trend of human diseases, it is recommended to add health communication as a unit of the curricula of the studies of media and communication/mass communication. So, in 1992 with this attention growing worldwide, department of mass communication of the University of Kelaniya, Sri Lanka adopted health communication into their curricula of Mass Communication. In this context, there is a de-

\footnotetext{
${ }^{*}$ Corresponding author: Mapa Thilakarathna

†Email: mapamc@kln.ac.lk
} 
velopmental aspect of conducting more and more filed and experimental researches on amalgamating both communication and health sciences.

Exploring the current nature of health communication systems and programs and creating a health communication program mitigating existing constraints and backdrops are my fundamental objectives of this current study. Polpithigama is a village of NorthWestern province in Sri Lanka, which is an interesting socio-anthropological place for developmental studies for many researchers as it remains in a flux of traditional socio-culture and modern social and economic modernization.

Fifty $(50)$ of subjects were equipped to this research while being majority $90 \%$ of Sinhala Buddhists as representing the general population in Sri Lanka, along with Tamil, Muslims, Burger, and other ethnicities. And, ten (10) in-depth interviews were used. As a background, there are some misconceptions of health behaviors, as this village is traditionally constructed by many myths and religious and cultural legends, and traditional forms of conventional habits and non-modern aspects of rural treatments for meditative illnesses among adults that is evolving through number of generations from decade to decade.

Therefore, it is believed that more Behavioral Change Communication (BCC) practices and guidelines could be immensely utilized to expel and mitigate the existing health risks and challenges among villages of this rural area. For example, dental diseases and how they do care about the dental aspects is one of the important questions of this context of lack of sufficient health knowledge in the respective areas of exploring in this current study. Charcoal and silt-sand are some of the tools that they used to brush their teeth in this village, and mostly, chewing Betle after dinner. Rural Sri Lankan people are used to keep it in their mouth without brushing their mouth, which are some of the health risks pervasively shown in this area.

Moreover, it is only after 1960's that there is scheduled system for vaccination and injection for child care medicine among kids and children in Sri Lanka. Not only that; even the teens who go to school were not having sufficient level of knowledge and education system even in school curricula on sexual and reproductive health sciences, so many teens were vulnerable to the issues of child marriages and premature born kids. In this background, it is needed to conduct a research on identifying such community level health risks and emerging health problems, and ultimately, it will make a pertinent plan of health communication under the spectrum of behavior change communication for getting such vulnerable communities back to effective health conditions from diseased and illness situation.

\section{REVIEW OF LITERATURE}

Schiavo [1] have drawn their further attention on Health Communication: From Theory to Practice. Especially, modern perspectives of ground level developmental programs of health communication are widely as well as critically analysed for theory and productive practical approaches.

Kyun et al. [2] described Health Communication as Strategies for Developing Global Health Programs, which is crucially significant to understand by both developed and developing nations in the age of new media and rocketing up the contexts of grass root level communications with new lateral strategies.

Thompson [3] in the Encyclopedia of Health Communication, contributed a broad spectrum of intersectional disciplinary approaches to health communication in the times of modern day implementation level. There are many geographical innovations and ground level developmental aspects were supported by the health communication theories. 
Graeff et al. [4] have made a substantial contribution on identifying realities of developing countries' health communication experiences with special references to the behavioral change communication with the amalgamation of recently identified communication knowledge and sciences.

De Fossard et al. [5] pointed out the crucial necessity of the Communication for Behavior Change: Using Entertainment Education for Distance Education, particularly in the context of new media culture and media popular entertainment experiences.

Immigrant populations are vulnerable to serious health disparities, with many immigrants experiencing significantly worse health outcomes, such as higher rates of morbidity and mortality, than other segments of society. Immigrants disproportionately suffer from heart attacks, cancer, diabetes, strokes, HIV/AIDS, and many other serious diseases. These health risks demand effective health communication to help immigrants recognize, minimize, and respond effectively to potential health problems. In this, he studied meeting the health literacy needs of immigrant populations [6, 7].

Effective communication tools that can be applied in health risks in multicultural health care setting in his book provides insights into the complexities of multicultural relations in health care and demystifies the many cultural influences on health and healthcare to achieve its ultimate goal - to help people get the most they can out of healthcare and facilitate the promotion of public health [8].

An article reviews key communication issues involved in the design of effective and humane e-Health applications to help guide strategic development and implementation of health information technologies. There is a communication revolution brewing in the delivery of healthcare and the promotion of health fueled by the growth of powerful new health information technologies. He conducted the development, adoption, and implementation of a broad range of new e-Health applications [9].

Bernhardt [10] has revived communication at the core of effective public health. In this, he says that years from now, we may remember the fall of 2001 as the traumatic period in our country's history when public health became visible throughout society. While the dust was still settling from the terrorist attacks and the country grappled with fears of anthrax in our mail, the American public learned what professionals have known for years: the health and security of our nation depend heavily on a robust public health system. An additional revelation from this turbulent time was the critical role of strategic management.

The public today has greater access to health information than at any previous time in human history. Every day, people are inundated, even bombarded, with an abundance of health information. Healthcare professionals provide advice, pharmacists dispense printed instructions, health educators distribute brochures, television and radio news shows broadcast stories about peoples' health and well-being, newspapers offer coverage of the latest findings from medical research, and the Internet delivers in the modern age [11]. In this accessing, understanding, and applying of health communication messages, the challenge of health literacy is fully explored.

McAuley et al. [12] conducted a research on social relations, physical activity, and well-being in older adults. In this, a randomized controlled trial was conducted to examine: (a) the effect of two physical activity modes on changes in Subjective Well-Being (SWB) over the course of a 12-month period in older, formerly sedentary adults ( $N=174$, $M$ age $=65.5$ years) and (b) the role played by physical activity participation and social support in changes in SWB over time. 
Participants were randomized into either an aerobic activity group or a stretching and toning group. Structural equation modeling was employed to conduct multiple samples.

McAuley et al. [13] explored physical activity, aging, and psychological well-being. In this, review examines the effects of exercise and physical activity on the psychological well-being of older adults. Unlike most of the literature in this area, this review focuses primarily on those psychosocial outcomes that are generally positive in nature. As well as considering the overall effects of physical activity, the roles of program length, subject sex, age, physical fitness, and measurement are considered. Overall, the results of the 38 studies reviewed are overwhelmingly positive, with the majority reporting positive associations.

Hornik [14] has given many aspects of Public health communication: Evidence for behaviour change. In this, health communication and the usable communication with the intention of behaviour change applications are substantially treated by this study. In addition, Gurman et al. [15] contributed to the effectiveness of m-Health behaviour change communication interventions in developing countries. As a systematic study, there are many dimensions overlooked in the perspective of behaviour change communication.

A meta-analysis of the effect of mediated health communication campaigns on behaviour change in the United States was conducted by a group of researches [16]. In this, there are number of new effective forms of communication application.

As Jinadasa $[17,18]$ pointed out there is a great deal of potentials in using traditional forms of communication in community development program, in that sense, they can be used in health programs also. In addition to that, there is a growing development and expansion in social media, especially Facebook, among teen and youth cultures. There are some effective communication influences that can be made by the use of new media forms [19] in addition to mass media, while traditional forms of communication remain much significant in development communication as well [20].

Jinadasa [21] elaborated in community empowerment program, the North-Western province remains much interesting aspect in order to understand and plan productive rural level development communication programs. Further, he envisaged Sri Lankan development perspective can be much developed by the use of recently emerging health risks and global perspective of development discourses and modern liberal non-Western development re- reading that is much influenced by the works of Arthuro Escobar's Encountering Development [22].

Pannilage [23] pointed out even in the aspects of Globalization and Construction of Local Culture in Rural Sri Lanka, there is a significance in the values and customs of local knowledge in rural development. Pannilage [24] in his Socio-economic Analysis on the Gender Wage Gap Among Agricultural Laborers in Rural Sri Lanka, further elaborated the importance of agricultural labors in health sectors as in early days, it was much instrumental in development of their family economy with sustainable capacities in the facets of nutrition, well-being, and maternity health sectors.

\section{RESEARCH METHODOLOGY}

This is a field survey of both quantitative and qualitative methods of research. Polpithigama is a village of North-Western province in Sri Lanka, which is an interesting socio-anthro pological place for developmental studies for many researchers as it remains in a flux of traditional socio-culture and modern social and economic modernization. 
Fifty (50) of the subjects were equipped to this research while being majority $90 \%$ of Sinhala Buddhists as representing the general population in Sri Lanka, along with Tamil, Muslims, Burgers and other ethnicities.

Hypothesis of my study is with these social statistics, it is difficult to find out a scientific method of health knowledge among vulnerable rural villages as they are traditionally bound with the conventional forms of myths and non-scientific methods of health protection practices.

Exploring the current nature of health communication systems and programs and creating a health communication program mitigating existing constraints and backdrops are my fundamental objectives of this current study. Polpithigama is a village of NorthWestern province in Sri Lanka, which is an interesting socio-anthropological place for developmental studies for many researchers as it remains in a flux of traditional socio-culture and modern social and economic modernization.

\section{FINDINGS AND DISCUSSION}

According to the field survey, majority $65 \%$ subjects were female while $35 \%$ were male.

\section{Percentage of Gender}

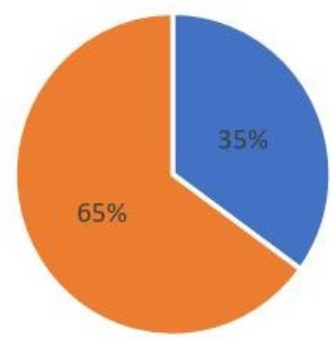

Female Male

FIGURE 1. Gender percentage

Majority of people are Sinhala Buddhist (98\%) when 2\% of participants are Muslims. Age of majority is from 21 to 30 (33\%), while from 31 to 40 age group is $22 \%$, and least numbers are $10 \%$ and $2 \%$ for $51-60$ and $61-70$, respectively.

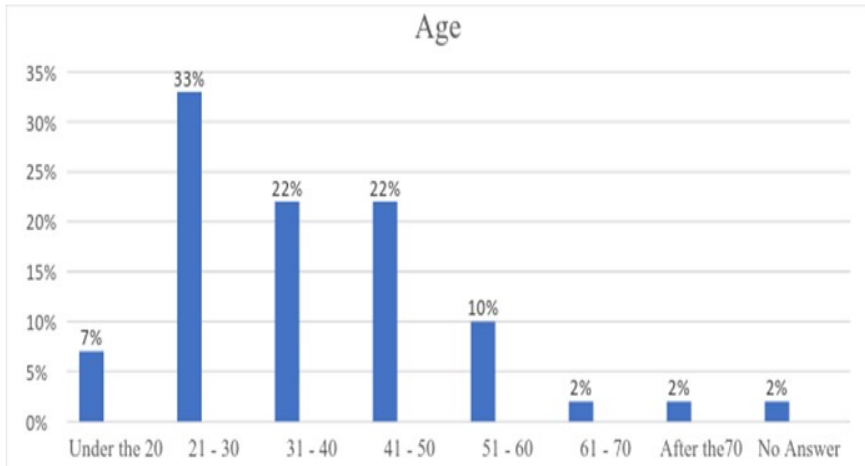

FIGURE 2 . Age 
When it is educational level of the sample, majority of them are Advanced Level pass is $59 \%$, while $37 \%$ are ordinary level pass people. It is only $2 \%$ of subjects who are university degree holders which is also significant as this sample reflects its minimal level of literacy in higher education at the university level.

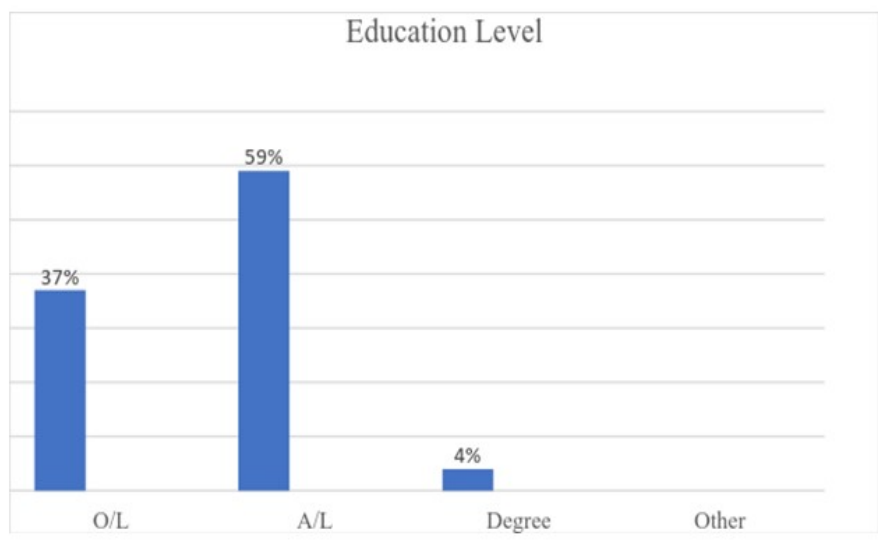

FIGURE 3 . Education level

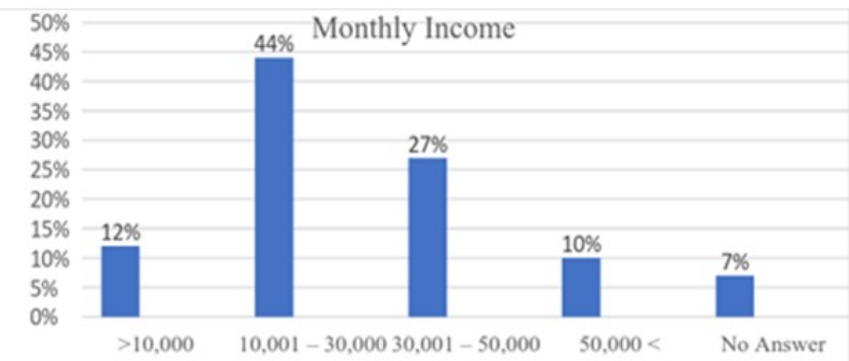

FIGURE 4 . Monthly income

Monthly income distribution can be shown as 10,001 to 30,000 , being the significant for most villagers as $44 \%$, while its monthly income is 30,001 to 50,000 , i.e., $27 \%$. The maximum is more than 50,000 , that is, only $10 \%$ of the subjects, reflecting the law economic poverty stricken in this area.

When it comes to the employment, $36 \%$ of the majority are self-employed based on local market and production system for many years, since they are used to embark on regional agricultural as well as other crops' cultivation and some of the small industrial employment at the domestic level.

Public sector employment is only $17 \%$ and majority of them are private sector, i.e., $28 \%$, while another $12 \%$ remain in jobless situation. It is interesting there is a $7 \%$ of statistic that was not answered by the questionnaire. It is also supposed to be jobless or illiterate section of the area analysis.

$66 \%$ people are married while it is only $20 \%$ who are unmarried when there is $2 \%$ of subjects who remain divorced by family and economic issues. 


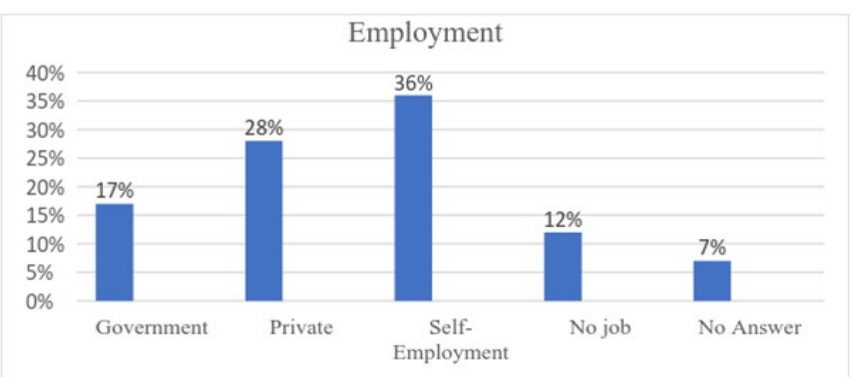

FIGURE 5 . Employment

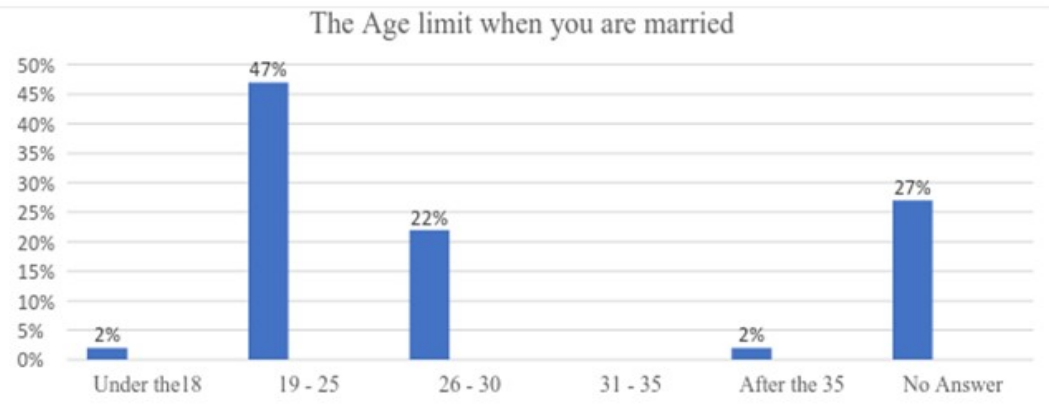

FIGURE 6. The age limit when you are married

After these kind of demographic data, it is concerned by the study problems gradually as first as the age limit when they are married. 47\% of majority are between 19 and 25 age limit when they are married, and 26-30 are $22 \%$, while under the eighteen remain only $2 \%$. It is significant that there is no one who married after the age of thirty.

Lots of them do not expect to have furthering their family by more future children, i.e., $43 \%$. When they use birth control method, it is only $39 \%$ using them properly while majority of $49 \%$ do not use them anywhere. Subjects were asked what are their birth control methods; $12 \%$ used condoms, while $7 \%$ used vaccine, $4 \%$ and $2 \%$ are tablets users and loop wearers, respectively. It is significant by maximum of $53 \%$ who did not answer this question, reflecting that they have no such scientific knowledge of birth controlling in this kind of rural Sri Lankan village, even in this modern digital media society and super popular cultural society.

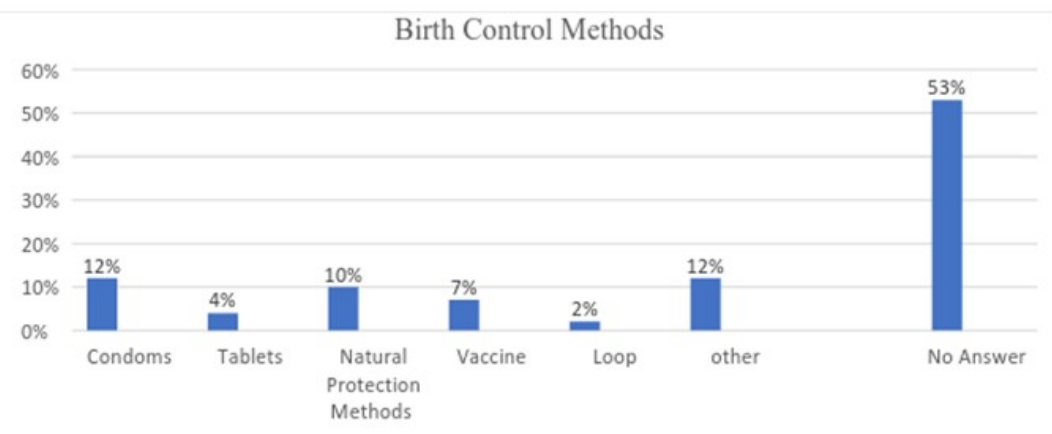

FIGURE 7. Birth control method 
Mid Wife is the major mode of health informational channel, while $7 \%$ and $2 \%$ are from parents and friends, respectively. It is significant that there is only $17 \%$ of the impact of media on health information provided in this area.

In Which way do you getting family Health Information

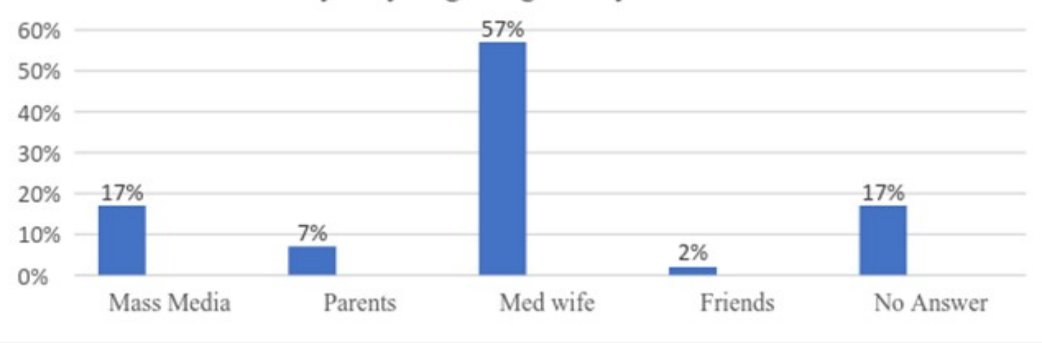

FIGURE 8. Family health information

$59 \%$ of subjects have responded that they are not medically treated for a long time and suffered from diseases. Only $2 \%$ did not answer while $39 \%$ are receiving long-term medical treatment for their chronic diseases. Diabetics and high blood pressure remain $12 \%$ of the majority of the diseases among subjects, while Kidney disease and Asthma remain $2 \%$ and $4 \%$, serially.

If Yes what are those?

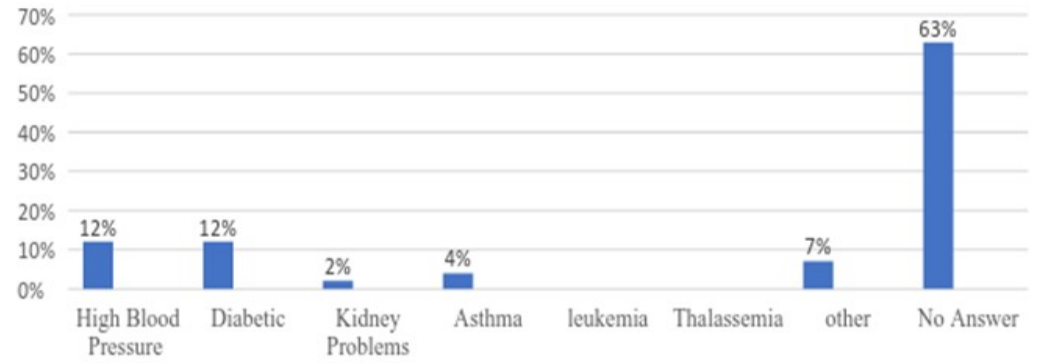

FIGURE 9. Demographic information of family health information

Even they live in traditional indigenous place, they are heavily used to take Western medicine (37\%), and only $4 \%$ are taking their local medicine of Ayurveda or Sinhala Indigenous medicine (Sinhala wedakama).

Most of the subjects used to brush their teeth two times per day (76\%), while only $17 \%$ are taking one-time teeth brush. Cleaning after meals is $71 \%$ while only $27 \%$ take it before the meals. Albeit, this area has pure water sanitation, but $44 \%$ are having drinking water issue, while $12 \%$ having it sometime, so that majority of them are suffering from drinking water issue. However, there is $44 \%$ (same portion) having no issue of drinking water. Most of the people take water from their wells (61\%) while some use common well (12\%). Tap line usage is only $7 \%$ users present in this current study.

In their daily medical treatment, most of them are used to go to government hospital (46\%), while another $15 \%$ are used to go to private hospital. It is interesting that $32 \%$ of them used to take their medicine from their own native doctors or perhaps the indigenous medical doctors.

When it comes to their daily food and nutrition, $98 \%$ of them have rice and curry as the major Sinhala meal for their breakfast, while only $2 \%$ are used to take bread. For din- 
ner, they are used to take rice and curry (96\%) while string hoppers remain 2\%. However, they are totally satisfied with their family health by $92 \%$ of a majority.

\section{CONCLUSION}

As this current study reported, it is not only the Western medicine but they use their native doctors or their traditional and indigenous medicine for their diseases.

It is only the mid-wife whose role has been key in health information in community health in their day to day life, while mass media play a minimal role in health communication in this area. Traditional forms of communication, such as indigenous forms of knowledge, can be used to spread the scientific health knowledge among the respective community.

As there is an issue in drinking water, respective authorities should take the necessary action based on the basis of community health organizations' recommendations, so that the use of indigenous knowledge can be productively implemented in further searching of alternative water resources in this area.

There should be lessons from the form of community-based information leaflets on relevant health risks, and how to take soluble action at the face of vulnerable situation on this context. Despite of having a program of health education for media by Health Education Bureau, it is vital to create community-based awareness campaign using their local literacy level, and it is very effective to make use of their own community level leaders to communicate most scientific knowledge in a realistic way.

Albeit, there are many health developmental programs conducted by World Health Organization in Sri Lanka, community health could be preserved by the use of understanding economic and political interferences that are vastly embedded in the far rural villages.

It is from both field survey and in-depth interviews. This study can be concluded that majority of the subjects were not sufficiently aware of their personal health and hygiene by the respective community health programs conducted in this area for many years. Though there were many rural development projects in this area for many years, community health issues of identifying their personal health matters, chronic diseases, reproductive health risks, and sexually transmitted diseases were not substantially addressed by the respective community development programs.

\section{REFERENCES}

1. Schiavo R. Health communication: From theory to practice. Hoboken, NJ: John Wiley \& Sons; 2013.

2. Kyun Kim D, Singhal A, Kreps GL. Health Communication: Strategies for developing global health programs. Bern, Switzerland: Peter Lang Publisher; 2014.

3. Thompson TL. Encyclopedia of health communication. London, UK: Sage Publications; 2014.

4. Graeff JA, Elder JP, Booth EM. Communication for health and behavior change: A developing country perspective. San Francisco, CA: Jossey-Bass Publishers; 1993.

5. De Fossard E, Bailey M. Communication for behavior change: Using entertainment-education for distance education. Mumbay, India: Sage Publications; 2016.

6. Kreps GL, Sparks L. Meeting the health literacy needs of immigrant populations. Patient Education and Counseling. 2008; 71(3): 328-32. DOI: 10.1016/j.pec.2008.03.001 
7. Kusoom W. Health behaviors and health risk behaviors among vocational students: Case study of vocational college in Nakornratchasima province Thailand. Journal of Advances in Health and Medical Sciences. 2016; 2(3): 102-96. DOI: $10.20474 /$ jahms2.3.4

8. Kreps GL, Kunimoto EN. Effective communication in multicultural health care settings. Thousand Oaks, CA: Sage Publications; 1994.

9. Kreps GL, Neuhauser L. New directions in e-Health communication: Opportunities and challenges. Patient Education and Counseling. 2010; 78(3): 329-336. D0I: 10.1016/j.pec.2010.01.013

10. Bernhardt JM. Communication at the core of effective public health. American Journal of Public Health. 2004; 94(12): 2051-2053. DOI: 10.2105/ajph.94.12.2051

11. Bernhardt JM, Cameron KA. Accessing, understanding, and applying health communication messages: The challenge of health literacy. In T. L. Thompson, A. M. Dorsey, K. I. Miller, R. Parrott, eds. Handbook of health communication. Mahwah, NJ: Lawrence Erlbaum Associates Publishers; 2003.

12. McAuley E, Blissmer B, Marquez DX, Jerome GJ, Kramer AF, Katula J. Social relations, physical activity, and well-being in older adults. Preventive Medicine. 2000; 31(5): 608-617. DOI: 10.1006/pmed.2000.0740

13. McAuley E, Rudolph D. Physical activity, aging, and psychological well-being. Journal of Aging and Physical Activity. 1995; 3(1): 67-96. DOI: 10.1123/japa.3.1.67

14. Hornik R. Public health communication: Evidence for behavior change. Abingdon, UK: Routledge; 2002.

15. Gurman TA, Rubin SE, Roess AA. Effectiveness of Health behavior change communication interventions in developing countries: a systematic review of the literature. Journal of Health Communication. 2012; 17(sup1): 82-104.

DOI: $10.1080 / 10810730.2011 .649160$

16. Snyder LB, Hamilton MA, Mitchell EW, Kiwanuka-Tondo J, Fleming-Milici F, Proctor D. A meta-analysis of the effect of mediated health communication campaigns on behavior change in the United States. Journal of Health Communication. 2004; 9(S1): 71-96. DOI: 10.1080/10810730490271548

17. Jinadasa WW. Community development programmes and folk-media: A communication model for Sri Lankan rural society. Global Media Journal-Indian. 2015; 2(2): 23-39.

18. Jinadasa M. Bali rituals and therapeutic communication in the traditional rural society in Sri Lanka. Journalism and Mass Communication. 2016; 6(11): 679-699. DOI: 10.17265/2160-6579/2016.11.003

19. Jinadasa M. Internet through mobile phone and its cognitive and behavioral impact: Changing sexuality. Journalism and Mass Communication. 2015; 5(12): 614-628. DOI: 10.17265/2160-6579/2015.12.002

20. Jinadasa M, Rajapaksha C, Pannilage U. A Sri Lankan development communication model: Use of traditional forms of communication. Journalism and Mass Communication. 2016; 6(8): 427-454. DOI: 10.17265/2160-6579/2016.08.001

21. Jinadasa MPK. Development communication; theory and practice. Colombo, Sri Lanka: Godage Intemational Publishers; 2011.

22. Jinadasa M. Newspaper reporting of suicide impact on rate of suicide in the contemporary society in Sri Lanka. Journalism and Mass Communication. 2016; 6(5): 237-255. DOI: 10.17265/2160-6579/2016.05.001

23. Pannilage U. Globalisation and construction of local culture in rural Sri Lanka. Sociology Study. 2016; 6(7): 448-461. DOI: $10.17265 / 2159-5526 / 2016.07 .003$

24. Pannilage U. A Socio-economic analysis on the gender wage gap among agricultural laborers in rural Sri Lanka. American Scientific Research Journal for Engineering, Technology, and Sciences. 2017; 30(1): 338-350. DOI: $10.3362 / 9781780440507.015$ 